\title{
GERENCIA DE RECURSOS HUMANOS EN LOS PROYECTOS APLICACIÓN DEL PRINCIPIO DE LA CARGA VERTICAL EN EL TRABAJO PARA EL DESARROLLO DEL EQUIPO DEL PROYECTO
}

\section{MANAGEMENT OF HUMAN RESOURCES IN THE PROJECTS APPLICATION OF THE PRINCIPLE OF THE VERTICAL LOAD IN THE WORK FOR THE DEVELOPMENT OF THE PROJECT TEAM}

\section{RESUMEN}

\author{
Luis Humberto Bravo Salomón ${ }^{\mathrm{a}}$
}

Dentro de las áreas del conocimiento del PMI se encuentra la Gestión de los Recursos Humanos, la misma que se encarga de los procesos que organizan y dirigen el equipo del proyecto, el mismo que está compuesto por personas con roles y responsabilidades asignados para concluir el proyecto. Teniendo en cuenta que es necesario contar con personal involucrado e identificado con el proyecto, es necesario conocer alguna técnica a aplicar para lograr ese objetivo.

El psicólogo Frederick Herzberg señala que existen tres filosofías generales de la administración de personal. La primera se basa en la teoría organizacional, la segunda en la ingeniería industrial, y la tercera en la ciencia de la conducta.

Dentro de esta última filosofía, Herzberg plantea lo que él denomina "Principios de la Carga Vertical de Trabajo", señalando que esto consiste en dar factores motivadores al asignar un trabajo. Para ello determina siete principios que los asocia, cada uno de ellos, a "tipos" de motivadores, que los denomina motivadores implicados. Con ello logra lo que el llama "Enriquecimiento Laboral".

Palabras clave: Gerencia de Proyectos, Gerencia de Recursos Humanos, Factor Humano, PM

\section{ABSTRACT}

Within the areas of the knowledge of the PMI one is the Management of the Human Resources, the same that is in charge of the processes that organize and direct the project team, the same that is made up of people with rolls and assigned responsibilities to conclude the project. Considering that is necessary to count on personnel involved and identified with the project, it is necessary to know some technique to apply to achieve that objective.

Psychologist Frederick Herzberg indicates that three general philosophies of the personnel administration exist. First one is based on the organizational theory, second in industrial engineering, and third in the science of the conduct.

Within this last philosophy, Herzberg he raises what it denominates "Principles of the Vertical Load of Work", indicating that this consists of employing to motivating factors when assigning. For it he determines seven principles associate that them, each of them, to "types" of motivating, denominate that them motivating implied. In this way he obtains what the flame "Labor Enrichment".

This theory appears, the same that has been applied of experimental way obtaining satisfactory results.

Key words: Management of Projects, Management of Human Resources, Human Factor, PMI

\section{OBJETIVOS}

En octubre del año 2004 se publica la Guía de los Fundamentos de la Dirección de Proyectos (Guía del PMBOK) Tercera Edición, Norma Nacional Americana ANSI/PMI 9-001-2004, la misma que reemplaza a la Edición 2000, que se publicó como Segunda Edición de la Guía del PMBOK.

El equipo del proyecto está compuesto por personas que cumplen determinadas funciones y tienen responsabilidades asignadas, con la finalidad de desarrollar y concluir el proyecto.

Este personal debe estar plenamente involucrado e identificado con el proyecto.

Para lograr este objetivo, podemos aplicar la teoría denominada "La Teoría de la Carga Vertical en el trabajo para el desarrollo del equipo del proyecto dando factores motivadores al asignar un trabajo.

\section{DESARROLLO}

\section{Marco teórico}

\section{El Equipo del Proyecto según la Guía del PMBOK - Tercera Edición -2004}

EI PMI señala que el Equipo del Proyecto hace referencia a todos los miembros del equipo del proyecto, incluidos el equipo de dirección del proyecto, el director del proyecto $y$, para algunos proyectos, el patrocinador del proyecto.

Cuando hablamos de los miembros del equipo del proyecto, nos referimos a las personas que dependen, ya sea directa o indirectamente, del director de proyectos. Ellos son los responsables de ejecutar el trabajo del proyecto como una de sus obligaciones normales asignadas.

\footnotetext{
a Ingeniero Civil - PUCP. Máster en Consultoría de Construcción.Universidad Politécnica de Madrid
} 
Aquí hay un término al cual el PMI le asigna una definición interesante: trabajo. Trabajo lo define, en su glosario, como el esfuerzo físico o mental, empleo o ejercicio de una habilidad en forma sostenida, para superar obstáculos y lograr un objetivo; definición que en su primera parte es muy similar a la primera acepción que podemos encontrar en el Diccionario de la Lengua Española. Sin embargo, le añade algo que es justamente lo que hace que ella se diferencie de cualquier otra actividad: empleo de una habilidad.

La habilidad es definida como la capacidad para usar los conocimientos, una aptitud desarrollada o una capacidad para ejecutar o realizar una actividad en forma eficiente y de inmediato.

Las habilidades interpersonales incluidas en la Guía son:

$>$ Comunicación efectiva. Intercambio de información.

$>$ Influencia en la organización. Capacidad para lograr que las cosas se hagan.

$>$ Liderazgo. Desarrollar una visión y una estrategia, y motivar a las personas a lograrlas.

$>$ Motivación. Estimular a las personas para que alcancen altos niveles de rendimiento y superen los obstáculos al cambio.

$>$ Negociación y gestión de conflictos. Consultar con los demás para ponerse de acuerdo o llegar a acuerdos con ellos.

$>$ Resolución de problemas. Combinación de definición de problemas, identificación y análisis de alternativas, y toma de decisiones.

La Gestión de los Recursos Humanos del Proyecto del PMI señala que existen cuatro procesos que organizan y dirigen el equipo del proyecto, uno de ellos es y que son la Planificación de los Recursos Humanos, Adquirir el Equipo del Proyecto, Desarrollar el Equipo del Proyecto y, Gestionar el Equipo del Proyecto.

Desarrollar el Equipo del Proyecto consiste en mejorar las competencias y la interacción de los miembros del equipo para lograr un mejor rendimiento del proyecto.

\section{COMENTARIOS AL MARCO TEÓRICO}

En el presente trabajo se mostrará una manera de realizar el desarrollo del equipo del proyecto basada en la teoría del Psicólogo Frederick Herzberg.

Para ello es necesario analizar, previamente, el proceso "Desarrollar el Equipo del Proyecto" (Proceso 9.3), con el cual se pretende mejorar las competencias e interacciones de los miembros del equipo a fin de mejorar el rendimiento del proyecto. En ese proceso se señala que los principales objetivos son:

$>$ Mejorar las habilidades de los miembros del equipo a fin de aumentar su capacidad de complementar las actividades del proyecto.

$>$ Mejorar los sentimientos de confianza y cohesión entre los miembros del equipo a fin de incrementar la productividad a través de un mayor trabajo en equipo.
Para lograr estos objetivos, es decir el desarrollo del equipo del proyecto, podemos relacionar lo que dice el Psicólogo Frederick Herzberg, distinguido profesor de Management en la Universidad de UTA autor del libro Work and the Nature of Man, quien señala que el logro, el reconocimiento, el trabajo en sí mismo, la responsabilidad, el avance y el crecimiento, son los factores que más afectan las actitudes en el trabajo.

Herzberg es el autor de la denominada "Teoría de los Factores", en la que señala que la motivación de las personas depende de dos factores y que, resumidamente, podemos decir que son:

a) La satisfacción en el cargo es función del contenido o de las actividades desafiantes y estimulantes del cargo: estos son los llamados factores motivadores. La insatisfacción en el cargo es función del ambiente, de la supervisión, de los colegas y del contexto general del cargo: son los llamados factores higiénicos.

b) Herzberg llegó a la conclusión de que los factores responsables de la satisfacción profesional están desligados y son diferentes de los factores responsables de la insatisfacción profesional: "lo opuesto a la satisfacción profesional, no es la insatisfacción, es no tener ninguna satisfacción profesional; de la misma manera, lo opuesto a la insatisfacción profesional es carecer de insatisfacción profesional y no la satisfacción".

Mientras Maslow elaboró una teoría de la motivación con base en el concepto de jerarquía de necesidades que influyen en el comportamiento humano y la sustenta, precisamente, en las diversas necesidades humanas (enfoque orientado hacia el interior), Herzberg basa su teoría en el ambiente externo y en el trabajo del individuo (enfoque orientado hacia el exterior).

Además, Herzberg señala que existen tres filosofías generales de la administración de personal. La primera se basa en la teoría organizacional, la segunda en la ingeniería industrial, y la tercera en la ciencia de la conducta.

Dentro de esta última filosofía, Herzberg plantea lo que él denomina "Principios de la Carga Vertical de Trabajo", señalando que esto consiste en dar factores motivadores al asignar un trabajo. Para ello determina siete principios que los asocia, cada uno de ellos, a "tipos" de motivadores, que los denomina motivadores implicados. Con ello logra lo que el llama "Enriquecimiento Laboral".

En el siguiente cuadro podemos apreciar los principios de la carga vertical de trabajo y sus motivadores implicados, haciendo notar que en la mayoría de estos últimos se encuentra la responsabilidad, el logro y el reconocimiento, además del crecimiento y el aprendizaje, los que no debemos dejar de lado. 


\begin{tabular}{|c|c|c|}
\hline \multicolumn{3}{|c|}{ Principios de la Carga Vertical de Trabajo } \\
\hline \multicolumn{2}{|r|}{ Principio } & \multirow{2}{*}{$\begin{array}{l}\text { Motivadores implicados } \\
\begin{array}{l}\text { Responsabilidad y logro } \\
\text { individual }\end{array}\end{array}$} \\
\hline A & $\begin{array}{l}\text { Eliminar algunos controles, dejando la } \\
\text { obligación de rendir cuentas }\end{array}$ & \\
\hline$B$ & $\begin{array}{l}\text { Aumentar la obligación de los individuos de } \\
\text { rendir cuentas de su propio trabajo }\end{array}$ & $\begin{array}{l}\text { Responsabilidady } \\
\text { reconocimiento }\end{array}$ \\
\hline C & $\begin{array}{l}\text { Proporcionar a cada persona una unidad } \\
\text { natural de trabajo completa (módulo, } \\
\text { división, área, etc) }\end{array}$ & $\begin{array}{l}\text { Responsabilidad, logro y } \\
\text { reconocimiento }\end{array}$ \\
\hline D & $\begin{array}{l}\text { Conceder autoridad adicional a los } \\
\text { empleados en sus actividades; libertad en } \\
\text { el trabajo }\end{array}$ & $\begin{array}{l}\text { Responsabilidad, logro y } \\
\text { reconocimiento }\end{array}$ \\
\hline$E$ & $\begin{array}{l}\text { Proporcionar los informes periódicos } \\
\text { directamente a los propios trabajadores, no } \\
\text { a través de sus supervisores }\end{array}$ & Reconocimiento interno \\
\hline $\mathrm{F}$ & $\begin{array}{l}\text { Introducir tareas nuevas y más dificiles no } \\
\text { realizadas previamente }\end{array}$ & Crecimiento y aprendizaje \\
\hline G & $\begin{array}{l}\text { Asignar tareas especificas y especializadas } \\
\text { a las personas, permitiéndoles convertirse } \\
\text { en expertos }\end{array}$ & $\begin{array}{l}\text { Responsabilidad, crecimiento y } \\
\text { avance }\end{array}$ \\
\hline
\end{tabular}

Estos principios de la carga vertical de trabajo los contrapone con la clásica forma de asignar la carga de trabajo, a la que denomina Carga Horizontal de Trabajo, mediante la cual se reduce el aporte personal de los empleados en vez de darles oportunidad de desarrollarse en sus labores habituales.

Finalmente, añade que existe una aplicación de enriquecimiento laboral en la que, luego de seis meses de aplicación, se lograron resultados satisfactorios: se elevó el indicador del índice de calidad de servicio, de un $50 \%$ a un $90 \%$; se incrementó el indicador de puntaje medio de reacción al trabajo, de $40 \%$ a $55 \%$. En ambos casos se compararon con equipos en los cuales no se habían realizado ningún tipo de cambios y la diferencia era notoria.

\section{EJEMPLO DE APLICACIÓN PRÁCTICA}

\section{Aplicación del principio de la Carga Vertical en el Trabajo para el Desarrollo del Equipo del Proyecto (mediante el Enriquecimiento Laboral):}

Se plantea la metodología empleada por el Psicólogo Frederick Herzberg en la experimentación exitosa señalada anteriormente, para el enriquecimiento laboral:

1. Seleccionar los trabajos en los que: a) la inversión en ingeniería industrial (los procesos) hace que los cambios no sean muy costosos, b) las actitudes son deficientes, c) la higiene está convirtiéndose en algo muy caro, y d) la motivación marcará una diferencian en el desempeño.

2. Abordar estos trabajos con la convicción de que pueden ser cambiados.

3. Hacer una reunión para crear una lista de cambios que podrían enriquecer los trabajos, sin tener en cuenta su viabilidad.

4. Revisar la lista y eliminar las sugerencias que impliquen higiene, en lugar de motivación real.
5. Revisar la lista en busca de generalidades como "darles responsabilidad" que rara vez son seguidas en la práctica. Esto podrá parecer obvio, pero las palabras de motivación nunca han desaparecido de las empresas, pero su sustancia ha sido racionalizada y organizada. Palabras como "responsabilidad", "crecimiento" y "desafío", por ejemplo, han hallado su espacio lírico en el discurso patriótico de todas las organizaciones.

6. Revisar la lista para eliminar cualquier sugerencia de carga horizontal.

7. Evitar la participación directa de los empleados cuyos trabajos van a ser enriquecidos. El trabajo va a cambiar, y será el contenido de ese trabajo lo que produzca la motivación, no las actitudes respecto de tener participación en el proceso o el desafío inherente a definir un trabajo.

8. En los intentos iniciales de enriquecimiento laboral hacer un experimento controlado. Elija al menos dos grupos equivalentes, uno, una unidad experimental en el que se introducen los cambios, y el otro, un grupo de control en el que no se introduce ningún cambio. Será necesario efectuar pruebas de rendimiento y de actitud laboral antes y después del ensayo a fin de evaluar la efectividad del programa de enriquecimiento del trabajo.

9. Prepararse para una baja en el desempeño del grupo experimental durante las primeras semanas. El cambio a un nuevo trabajo puede llevar a una reducción temporal de la eficiencia.

10. Esté preparado para que algunos empleados muestren ansiedad y hostilidad hacia los cambios que usted propone.

\section{CONCLUSIONES}

Con relación a la aplicación del principio de la Carga Vertical en el Trabajo para el Desarrollo del Equipo del Proyecto, es necesario tener en cuenta algunas importantes consideraciones:

> No siempre debemos esperar que el resultado inicial sea del todo satisfactorio. Podemos tener, inicialmente, resultados un poco desalentadores, pero es de esperar que con el tiempo podamos lograr el éxito esperado.

$>$ Esta premisa anteriormente descrita se debe a que es importante recordar que los cambios requieren de un periodo de maduración.

> Considerando que es un método que está aún en proceso de maduración, además de ser una metodología aplicada en personas, consideramos importante señalar que siempre habrá un grado de incertidumbre en los resultados que obtengamos, por lo que se requiere de una mayor aplicación para determinar de una manera más cercana su grado de confiabilidad. 


\section{REFERENCIAS BIBLIOGRÁFICAS}

[1]. Guía de los Fundamentos de la Dirección de Proyectos (Guía del PMBOK) Tercera Edición - 2004, Norma Nacional Americana ANSI/PMI 9-001-2004

[2]. Construction Extensión to a Guide to the Project Management Body of Knowledge - PMBOK Guide - 2000 Edition

[3]. Administración de Recursos Humanos - R. Wayne Mondy - Robert M. Noe - ISBN - 968-880-949-7

[4]. Administración de Recursos Humanos - Adalberto Chiavenato - ISBN - 958-41-0037-8

[5]. Una vez más: ¿cómo motiva a sus empleados? - Frederick Herzberg - Artículo

[6]. Management y Alta Gerencia - Teoría de Mazlow y Teoría de Herzberg http://www.econolink.com.ar/managemen/manage2.htm

[7]. Diccionario de la Lengua Española - Vigésima segunda edición - ISBN: 84-670-0039-2 (O.C.)

[8]. El Factor Humano y el Éxito en la Gerencia de Proyectos - Luis Bravo - 2003 - ISBN: 84-930700-7-6

E-mail: bravo.Ih@pucp.edu.pe 